\title{
Bringing Atomic and Nuclear Physics Laboratory Data Into the Classroom
}

Eric B. Norman ${ }^{1}$, Ruth-Mary Larimer ${ }^{1}$, Gregory Rech $^{1,2}$, Jeffrey Lee ${ }^{1,3}$, Chue Vue ${ }^{1,4}$

\author{
Tholoana Leubane ${ }^{1,5}$, Kenneth Zamvil ${ }^{1,6}$, and Laura Guthrie ${ }^{1,7}$ \\ ${ }^{1}$ Lawrence Berkeley National Laboratory, Berkeley, CA \\ ${ }^{2}$ University of California, Berkeley, CA \\ ${ }^{3}$ University of California, Irvine, CA \\ ${ }^{4}$ California State University, Fresno, CA \\ ${ }^{5}$ Franklin Middle School, Vallejo, CA \\ ${ }^{6}$ Hogan High School, Vallejo, CA \\ ${ }^{7}$ Acalanes High School, Lafayette, CA
}

\section{Contact:}

Eric B. Norman: Senior Staff Physicist, LBNL, Mail Stop 50R5008, Berkeley, CA 94720

e-mail: ebnorman@lbl.gov

Phone: 510-486-7846

Fax: 510-486-6738 


\title{
Bringing Atomic and Nuclear Physics Laboratory Data Into the Classroom
}

\begin{abstract}
To illustrate a number of basic concepts in atomic and nuclear physics, we have developed three websites where students can analyze data from modern laboratories. By working through the on-line procedures, students will become acquainted with characteristic x-ray spectra, the concept of half-life, x-ray fluorescence, and neutron activation analysis.
\end{abstract}

\section{Atomic and Nuclear Physics Standards}

At both the national and state levels, many educational organizations have adopted standards that call for the teaching of atomic and nuclear physics in the high school classroom. These standards include the basic concepts of atomic structure, nuclear structure, and radioactivity. While many textbooks cover these topics, in a highschool laboratory setting it is often difficult to perform experiments to illustrate these ideas. In order to address this issue, we have developed three websites where students can analyze atomic and nuclear physics data from modern laboratories. By working through the procedures described on these sites, students can gain familiarity with the 
concepts of characteristic x-ray spectra, half-life, and the techniques of x-ray

fluorescence, and neutron activation analysis.

\section{Viewing the Periodic Table with $x-$ rays}

X-rays are high-energy photons that are produced when electrons make transitions from one atomic orbit to another. If you send a photon into an atom with energy greater than the binding energy of an electron in that atom, the photon can knock that electron out of its orbit, leaving a hole (or vacancy). This hole can then be filled by another electron in the atom falling from a higher orbit, giving off an x-ray in the transition to conserve energy. This process is known as fluorescence. Each element in the periodic table has its own unique set of characteristic x rays. The allowed energies of electrons in atoms are discrete. These energies depend on two quantum numbers, called $\mathrm{n}$ and $l$. The principle quantum number, $n$, is an integer having values of $1,2,3 \ldots$ The orbital angular quantum number, $l$, is an integer having values of $0,1, \ldots, n-1$. Barkla assigned the letter $\mathrm{K}$ to electrons in the $\mathrm{n}=1$ shell, $\mathrm{L}$ to electrons in the $\mathrm{n}=2$ shell, and so on ${ }^{1}$. Electron transitions to the $\mathrm{K}$ shell of an atom are called $\mathrm{K}$ x-rays, and transitions to the $\mathrm{L}$ shell are called L x-rays. This historical nomenclature is still in use today.

We used an ${ }^{241}$ Am source, which emits a 59.5-keV gamma ray ${ }^{2}$, to fluoresce the elements. With this gamma ray we were able to fluoresce the $\mathrm{K}$ electrons of the elements ranging from $\mathrm{Ca}$ (atomic number, $\mathrm{Z}=20$ ), whose $\mathrm{K}$ electron has a binding energy of 4.038 $\mathrm{keV}$, to $\operatorname{Tm}(\mathrm{Z}=69)$, whose $\mathrm{K}$ electron has a binding energy of $59.390 \mathrm{keV}$. For elements with $Z$ ranging from 70 (ytterbium) to 83 (bismuth) and for thorium $(Z=90)$ and uranium $(Z=92)$, we were able to fluoresce only the $L$ shell electrons. To measure the $x-$ 
rays emitted from each target, we used a planar germanium detector sitting at a $90^{\circ}$ angle from the ${ }^{241} \mathrm{Am}$ source. We acquired the x-ray spectra in 512 channels using an ORTEC PC based data acquisition system. The energy spectrum shown in Figure 1 of germanium is a good example of one of our fluoresced elements. You can see two of the x-ray energy peaks caused by electron transitions to its $\mathrm{K}$ shell. The L x-rays of germanium are too low in energy to be seen with our detector. We produced a total of 63 energy spectra of individual elements plus 12 spectra of "unknown" samples that students can analyze in order to determine their elemental compositions. These spectra have been placed on the web at http://ie.lbl.gov/xray.

\section{What is a half life?}

The vast majority of the approximately 3000 known isotopes are radioactive. The half-life is the amount of time it takes for one-half of the nuclei in a sample to decay. It is abbreviated as $t_{1 / 2}$. Of the original nuclei present at $t=t_{0}$, half will decay if we wait one half-life, leaving one-half of the original sample after a total time of one half-life. After two half-lives, one-quarter of the original sample will remain and so on. Measured half-lives vary from tiny fractions of seconds to billions of years, depending on the isotope.

The number of nuclei in a sample that will decay in a given interval of time is proportional to the number of nuclei in the sample. This condition leads to radioactive decay showing itself as an exponential process. The number, $N$, of the original nuclei remaining after a time $t$ from an original sample of $\mathrm{N}_{0}$ nuclei is

$$
\mathrm{N}=\mathrm{N}_{0} \mathrm{e}^{-\lambda t},
$$


where $\lambda$ is the mean lifetime of the radioactive nuclei. From this relation, it can be shown that $t_{1 / 2}=0.693 / \lambda$. The isotopes we chose for this project beta minus decay into more stable isotopes. This beta minus decay often leaves the new isotope in an excited energy level, which in turn causes this new isotope to gamma decay down to its ground state (lowest energy state). These gamma rays (photons with energies usually higher than those of $\mathrm{x}$ rays) are characteristic for each isotope.

To measure the gamma rays emitted from each isotope, we used a coaxial germanium detector $5 \mathrm{~cm}$ thick and $5 \mathrm{~cm}$ in diameter sitting directly in front of the isotope sample. We acquired the gamma ray spectra in 4096 channels using an ORTEC, PC based data acquisition system. A typical gamma ray spectrum is displayed in Figure 2. Data were accumulated in sequential energy spectra whose durations were chosen on the basis of the half-life of each isotope. Students can analyze these spectra on-line to determine the peak areas as a function of time. Instructions are provided that describe the steps needed to extract the half-lives of the isotopes. An example of the kind of decay curve one can obtain from this data is shown in Figure 3. All of the gamma ray spectra that we acquired for this project can be found at http://ie.lbl.gov/gamma.

\section{Neutron Activation Analysis}

Neutron activation analysis (NAA) is a useful technique for determining the elemental composition of materials. NAA can determine the concentration of several different elements within a single sample of a material regardless of their chemical form 
or oxidation state. Because of its sensitivity and precision, NAA is widely performed in many different fields of science.

NAA is usually performed with neutrons from a nuclear reactor. In our case, we used neutrons produced by a mixed source of ${ }^{241} \mathrm{Am}$ and ${ }^{9} \mathrm{Be}$. Inside this source the following reaction takes place: ${ }^{9} \mathrm{Be}+{ }^{4} \mathrm{He} \longrightarrow{ }^{12} \mathrm{C}+{ }^{1} \mathrm{n}$, where ${ }^{1} \mathrm{n}$ is the neutron that is produced by the reaction. The most common type of neutron-induced reaction is the neutron-capture reaction. When a neutron fuses with the nucleus, a compound nucleus forms in an excited state that quickly decays to the ground state through emission of one or more gamma rays. The resulting nucleus is often radioactive and will beta decay to an excited state of another nucleus resulting in the emission of one or more characteristic gamma rays.

The samples of materials used for this project were placed on top of the neutron source in a shielded container for about 48 hours. To measure the gamma rays emitted from each isotope, we used a coaxial germanium detector $5 \mathrm{~cm}$ thick and $5 \mathrm{~cm}$ in diameter sitting directly in front of the sample. We acquired the gamma ray spectra in 4096 channels using an ORTEC, PC based data acquisition system. We used lead bricks to shield the detector from outside sources.

Bananas are known to be good nutritional sources of potassium, and the spectrum shown in Figure 4 obtained following the neutron activation of a banana proves that this is, indeed, the case. The peak at $1461 \mathrm{keV}$ is produced by the decay of the long-lived naturally occurring isotope ${ }^{40} \mathrm{~K}$. The peak at $1525 \mathrm{keV}$ is produced by the decay of ${ }^{42} \mathrm{~K}$ that results from neutron capture on the stable isotope ${ }^{41} \mathrm{~K}$. All of the data that we collected and the procedures needed to analyze them can be found at http://ie.lbl.gov/naa. 


\section{Conclusions and Acknowledgments}

By going through the analyses of the data available on the three websites described here, a number of basic concepts of atomic and nuclear structure and radioactive decay should become less abstract and easier to comprehend. We hope that these websites will be useful tools for students and teachers alike to become more familiar with atomic and nuclear processes. This work was supported by the U.S. Department of Energy under contract number DE-AC03-76SF00098.

\section{References}

1. R.G. Lerner, G.L. Trigg, Encyclopedia of Physics, Second Edition (VCH Publishers, inc., 1990), pp. 1366-1367

2. S. Y. F. Chu, L. P. Ekstrom, R. B. Firestone, $W W W$ Table of Radioactive Isotopes, http://ie.lbl.gov/toi.

3. For more information on nuclear science, see http://www.lbl.gov/abc 


\section{Figure Captions}

Figure 1. An x-ray energy spectrum of germanium showing its characteristic K x-ray energy peaks.

Figure 2. A gamma-ray energy spectrum of ${ }^{140}$ La displaying all three of its major gamma-ray peaks.

Figure 3. The decay curve of the $1596-\mathrm{keV}$ gamma ray from the decay of ${ }^{140} \mathrm{La}$. The half-life determined from this data is 1.69 days, which agrees quite well with the known value of 1.678 days $^{2}$.

Figure 4. A gamma-ray energy spectrum of a neutron-activated banana. The peak at $1461 \mathrm{keV}$ is produced by the decay of the long-lived naturally occurring isotope ${ }^{40} \mathrm{~K}$. The peak at 1525 $\mathrm{keV}$ is produced by the decay of ${ }^{42} \mathrm{~K}$ that results from neutron capture on the stable isotope ${ }^{41} \mathrm{~K}$. 


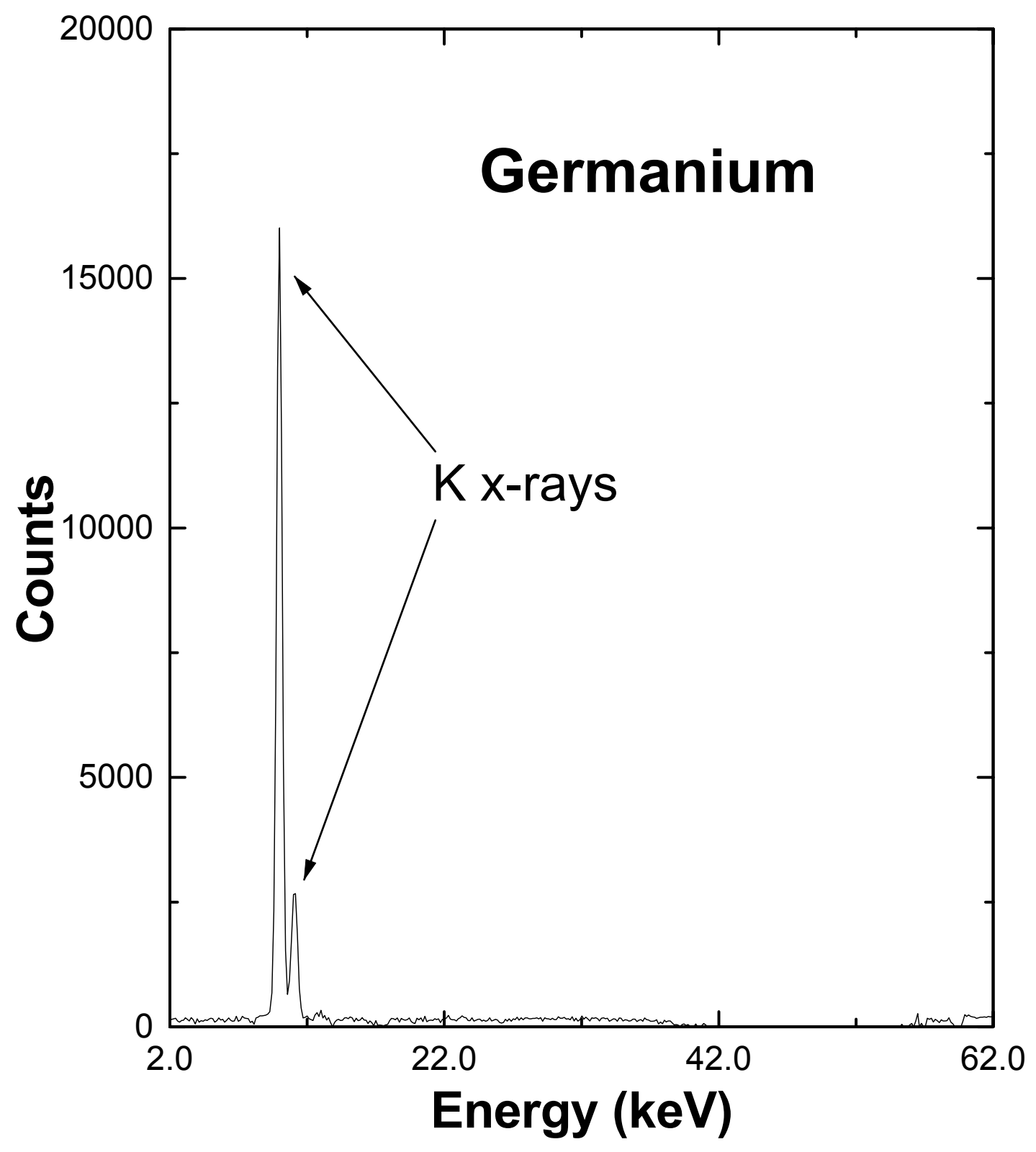

Figure 1 


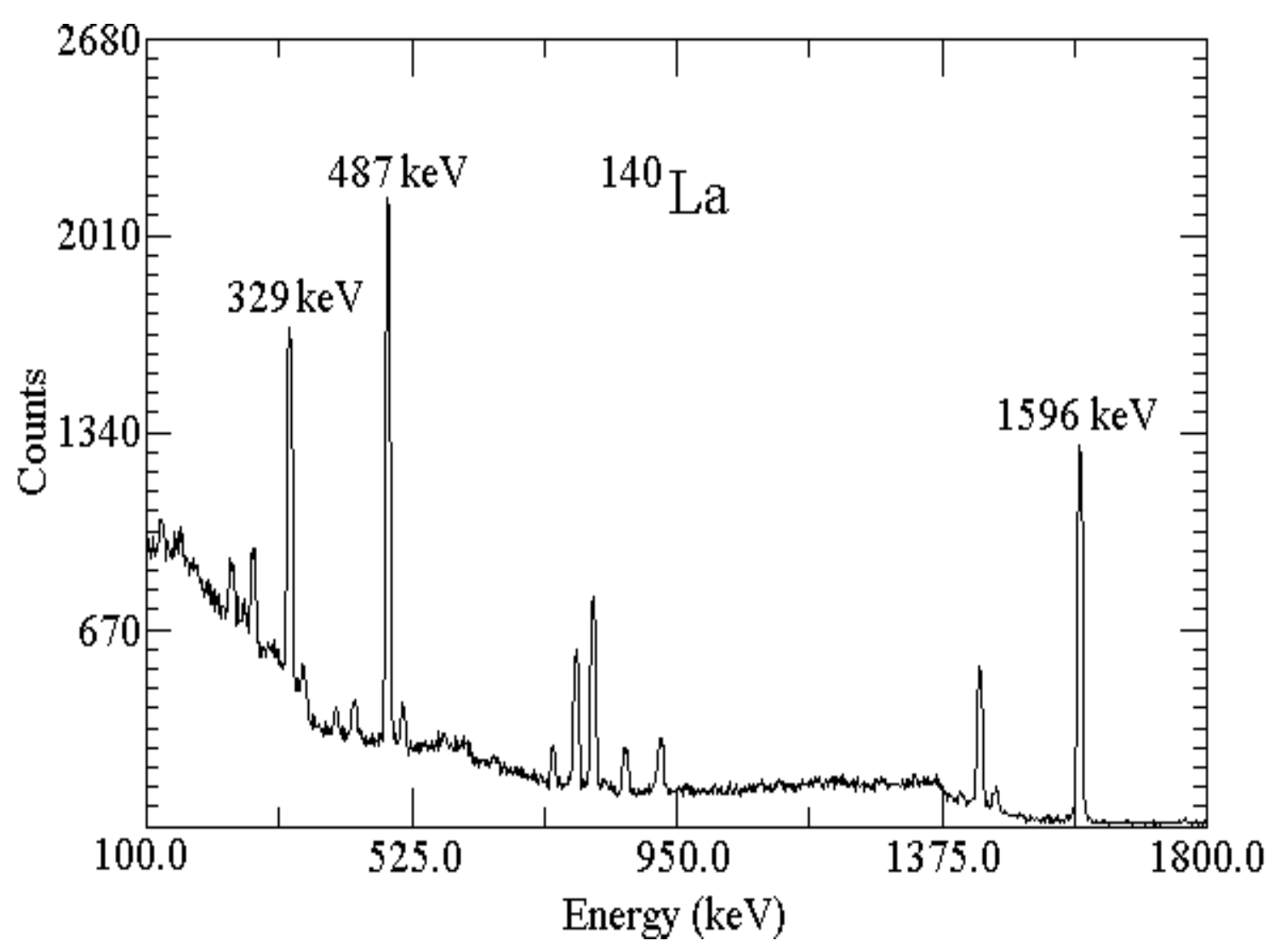

Figure 2 


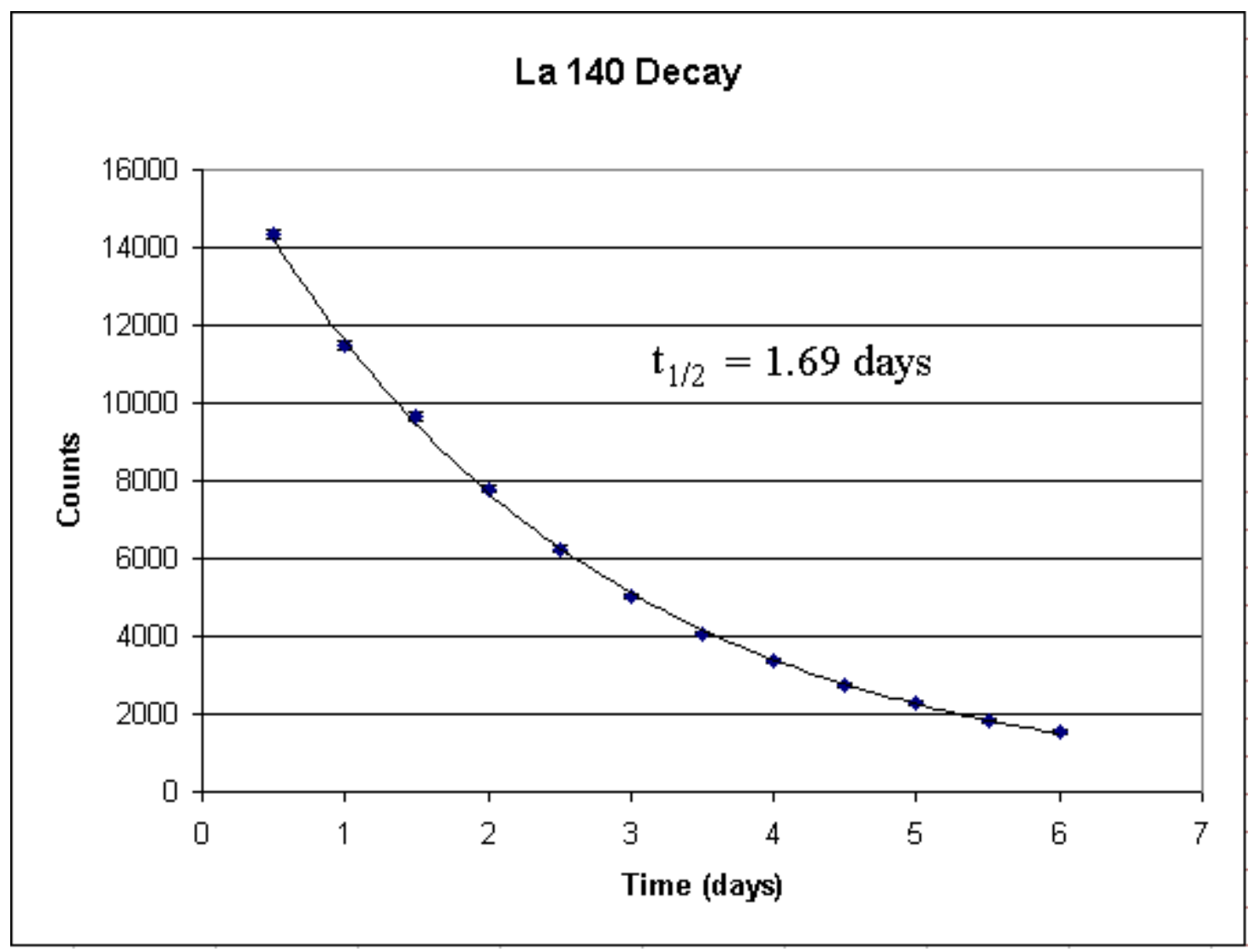

Figure 3 


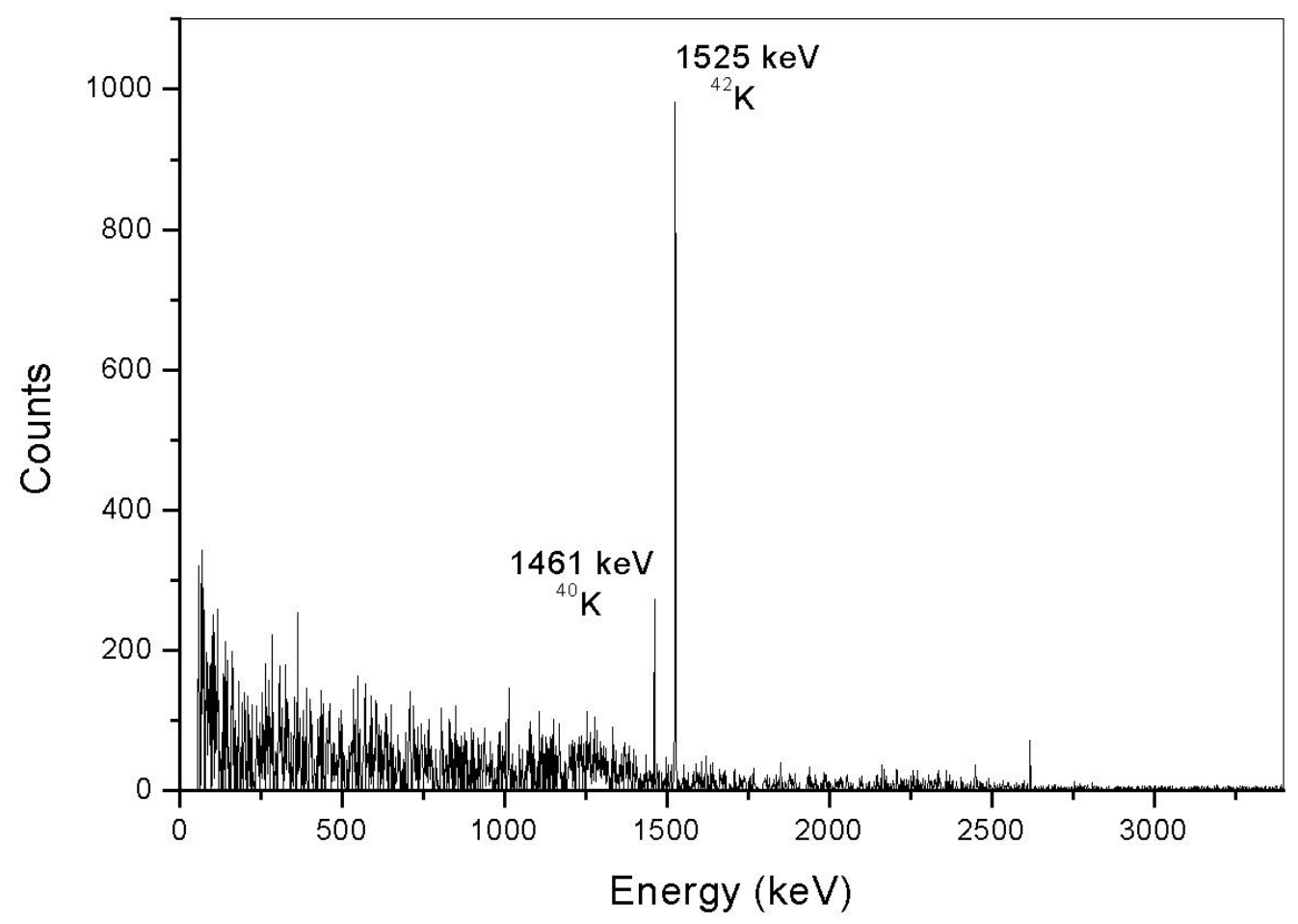

Figure 4 